\title{
Nutrition support teams: an integral part of developing a gastroenterology service
}

Reports of the incidence of malnutrition in hospital patients in the USA and UK started to appear in the 1970s. ${ }^{12}$ Since then the clinical importance of this finding has been clearly shown in many papers reporting an increased rate of complications of treatment and prolonged hospitalisation in malnourished patients. ${ }^{34}$ On the other hand artificial nutritional support in malnourished patients has been shown to reduce morbidity, mortality, and hospital stay. ${ }^{5-7}$ With increasing emphasis on health economics, these findings have subsequently been translated into an economic model, which carefully calculated the considerable, and potentially avoidable, added costs incurred by the hospital when treating undernourished patients. ${ }^{8}$ From the patient's perspective improvements in quality of life have been recorded with the introduction of nutritional support where indicated. ${ }^{9}$ Despite these reports, however, the recognition and treatment of malnutrition in 1995 remains an important problem in hospitals. ${ }^{10}$ The reason for this deficiency in properly assessing malnutrition may lie in the fact that clinical nutrition is not recognised as a clinical specialty and subsequently contributes poorly to undergraduate and postgraduate teaching programmes. A blueprint for the way forward was provided by the Kings Fund Centre Report in 1992, A Positive Approach to Nutrition as Treatment. ${ }^{11}$ The report highlighted the beneficial role to be played by nutrition support teams (NSTs) in the delivery of artificial nutritional support; however, only $37 \cdot 3 \%$ of hospitals in the UK have access to an NST in $1994 .^{12}$ As medical treatment improves in areas such as oncology and transplantation surgery, it is increasingly likely that patients will be vulnerable to the development of malnutrition. Suboptimal nutritional status could compromise the efficacy of the newly emerging treatments and this finding firmly underpins the need to improve the awareness and treatment of undernutrition, which can be best achieved by an NST.

No consensus has emerged as to the composition of such NSTs and great diversity in the number and professional origin of team members has been noted. ${ }^{13}$ In practical terms, however, the development of a nutrition advisory team or of the more active nutrition support teams is likely to involve an interested gastroenterologist, especially in centres without recall to academic laboratory facilities and research interests. Moreover, patients with small bowel Crohn's disease or intestinal failure are probably under the care of the gastroenterologist. In addition, insertion of percutaneous endoscopic gastrostomy and jejunostomy feeding tubes, and the prescription of nutrient delivery are often part of the remit of the gastroenterology team. As the specialty continues to develop, clinical nutrition is poised to play an ever increasing part in the delivery of a gastroenterology service, and it would seem timely to review the case for and against the formation of NSTs.

\section{Advantages of NSTs}

In view of the rapid expansion of available nutrient solutions, modes of administration, and the necessary monitoring, the NST is invaluable in ensuring costeffective and appropriate use of nutritional support. The support team is well positioned not only to assess the suitability of nutritional support for individual patients, but also to help avoid difficult ethical dilemmas, by offering objective specialised assessment to identify patients who will derive benefit from nutritional support, from those where a more conservative approach would be more appropriate. In cases where treatment is required the NST is designed to tackle the specific nutritional needs of the individual patients. Given the rapid expansion of knowledge in all areas of medicine it seems unlikely that individual practitioners from other specialties could accrue the necessary additional knowledge and experience in a short period of time to ensure optimum delivery of nutritional support. The NST also has the advantage of being a multidisciplinary team with access to all aspects of clinical nutrition, which can then be focused on the nutritional needs of individual patients. The benefits of this individual approach are substantiated by reports of a significant improvement in the standards of monitoring of patients receiving nutritional support and importantly in the rate of achieving nutritional goals when the NST is involved in patient care. ${ }^{14}$

Despite the considerable advances in nutritional support over the past 20 years, potentially life threatening complications still remain, particularly with parenteral nutrition. There is considerable evidence that NSTs can reduce the incidence of mechanical, infective, and metabolic complications associated with nutritional support. ${ }^{15} 16$ As well as the clear benefits to patient care the Kings Fund Centre Report also estimated that up to $£ 80000$ per annum per hospital could be saved from a reduction of septic complications alone. The main platform for such improvements in patient care stems from the implementation of standardised care protocols by NSTs that encourage attention to fine detail with all aspects of care for the malnourished patient. The argument that improvements in nutritional support can be realised through the introduction of care protocols alone would seem intrinsically flawed in view of the evidence highlighting the lack of recognition of malnutrition on general medical wards in the first instance. ${ }^{10}$ Furthermore when complications occur they may be life threatening. Catheter related infection and central vein thrombosis during parenteral nutrition may present in an atypical fashion, necessitating prompt recognition and careful treatment by an experienced team. The coordinated implementation of protocols by the NST also offers further advantages by facilitating data collection and providing an accessible basis to carry out clinical audit of the provision of nutritional support.

The formation of NSTs also provides the springboard to redress the deficiencies in the tuition of clinical nutrition. The multidisciplinary nature of the team facilitates and expedites the dissemination of knowledge to all relevant branches of the profession. Initially members of the team could fulfil a vital didactic role in their areas at both 
undergraduate and postgraduate level in terms of formal theoretical instruction and in the clinical setting, dealing with day to day practicalities. This last aspect will assume greater importance as the utilisation of enteral feeding increases in different medical specialties. Furthermore, as the profile of clinical nutrition increases, it will probably attract more funding for research, and the NST would seem the most qualified to keep abreast of developments and aid the translation of research finding to clinical practice. This aspect is highlighted by the increasing use of nutrients for pharmacomodulation, for example, the use of antioxidant vitamins and polyunsaturated fish oils to modulate the immune response. ${ }^{17}$ The role of NSTs at the interface between commercial provision and clinical use of nutritional solutions also places it ideally for supervising clinical trials to evaluate progress in clinical nutrition. On a more pragmatic plane, this position also affords the NST a more powerful role in negotiating the purchase of nutritional feeds from the commercial sector.

As clinical nutrition develops there are increasing numbers of patients who receive both enteral and parenteral nutritional support at home. This management step eases hospital expenditure and boosts patient morale. The instruction required in the first instance, the delivery of supplies on a regular basis, essential monitoring and 24 hour back up are most appropriately carried out by a dedicated NST. Patients feel more secure with home treatment if they know they have easy access to information and medical support. This provision of support is enhanced if the patient knows exactly where to turn for advice, rather than having to speak to the doctor on call, who is unlikely to be familiar with the practical problems of nutritional therapy. Patient support groups are also adding weight to the calls for an increase in the number of nutrition support teams. ${ }^{18}$

\section{The case against NSTs}

The evidence in favour of the establishment of NSTs would seem considerable; however, only $37 \cdot 3 \%$ of hospital units in the UK have access to such teams, suggesting either a large degree of reticence to dedicate the necessary funds, or that the medical community is still not prepared to recognise the prevalence of malnutrition and importance of instituting effective treatment. In support of the first explanation, it could be argued that nutrition is so fundamental to patient care that all clinicians should be familiar with nutritional assessment and the delivery of nutritional support. The acquisition of such knowledge and skills would be part of his or her continuing medical education programme. Furthermore, the cost-effectiveness of NSTs has been based on the reduction of serious complications associated with nutritional support. It could be argued, however, that the incidence of complications can be reduced by implementing care protocols and treatment algorithms without the extra expense of forming a nutrition support service. Physicians with an interest in clinical nutrition would act purely in a consultative manner in cases of extreme complexity. Interestingly, one report suggests that complications are reduced in this setting but complications are even fewer where there is more active involvement of a nutrition team. ${ }^{19}$ Another consideration is whether selective bias has been introduced when presenting the areas of cost saving due to the clinical impact of the support group. Patients receiving total parenteral nutrition are an important drain on hospital funds and any potential saving from a reduced complications rate seems very attractive to accountants and emphasises a group of patients where administrators could expect to see a large reduction in cost. Such patients requiring nutritional support, however, are also likely to be hospitalised for a long period of time for concomitant treatment and any complications arising from nutritional support therapy will be treated within that time scale; therefore, the financial argument that hospitalisation will be shortened by NSTs may not withstand closer scrutiny.

In the face of a growing awareness of malnutrition, the NST may become in the initial stages a victim of its availability, taking on an ever increasing number of patients, whom will receive nutritional support often from the community setting. Essentially important health benefits would be felt in the community with the hospital system funding the cost. This situation would be difficult to reconcile with the emerging fiscal system of health care delivery in the UK. The way forward would be to lay emphasis on the didactic role of the NST so that in time malnourished patients could be assessed and treated independently. As with all support groups and departments, to maximise its effectiveness an NST would need to be adequately resourced in terms of time, facilities, staff, and finance to supervise home and hospital care. Roles within the team have to be clearly defined and subject to re-evaluation once established. As a result the reasons that over $60 \%$ of hospitals do not have access to a nutrition support group may be that there is insufficient time and money to establish such a service. In addition, clinicians, nurses, and dietitians already established in the unit may resent colleagues becoming involved in the treatment of their patients, leading to difficulties in the working relationships.

The evidence is accumulating that nutrition support groups have a significant part to play in the improvement of patient treatment and in the provision of cost-effective health care. A mixture of financial, educational, and political factors may explain the delay in implementation of the proposals of the Kings Fund Centre Report. In many hospitals there is a shortage of suitably trained staff who could develop such a service, and clearly facilities will be required for the necessary education of staff before an NST can be established. Many of the theoretical reservations as to the efficacy of support groups may be overcome by thorough clinical studies looking at defined end points, such as the time required for improvements in quality of life and functional integration into society, rather than the incidence of complications as an inpatient. In the face of such a rapid expansion of medical knowledge and therapeutic intervention it is surely unacceptable not to tackle the problem of malnutrition in our patients, rather than leave it another 20 years.

N REYNOLDS J P MCWHIRTER C R PENNINGTON

Department of Clinical Pharmacology,

Ninewells Hospital and Medical School,

Dundee DD1 $9 S Y$

Correspondence to: Dr C R Pennington.

1 Bistrian RB, Blackburn GL, Vitale J, Cochran D, Naylor J. Prevalence of malnutrition in general medical patients. $\mathcal{F} A M A$ 1976; 235: 1567-70.

Weinsier RL, Hinker EM, Krumdieck CL, Butterworth CL. A prospective evaluation of Am $\mathfrak{f}$ Clin Nutr 1979; 32: 418-26.

3 Mullen JI Gertner MH, Buzby GP, Goodhart GL, Rosato EF. Implications of malnutrition in the surgical patient. Arch Surg 1979; 114: 121-5.

4 Symreng T, Anderberg B, Kagedal B, Norr A, Schildt B, Sjodahl R. Nutritional assessment and clinical course in 112 elective surgical patients. Acta Chir Scand 1983; 149: 657-62.

5 Bastow MD, Rawlings J, Allison SP. Benefits of supplementary tube feeding after fractured neck of femur: a randomised controlled trial. $B M \mathcal{F}$ 1983; 287: 1589-92.

6 Delmi M, Rapin CH, Bengoa JM, Delmas PD, Vasey H, Bonjour JP. Dietary supplementation in elderly patients with fractured neck of femur. Lancet 1990; 335: 1013-6.

7 Larsson J, Unosson M, Ek AC, Nilsson L, Thorslund S, Bjurulf P. Effect of dietary supplement on nutritional status and clinical outcome in 501 geriatric patients - a randomised study. Clin Nutr 1990; 9: 179-84.

8 Reilly JJ, Hull SF, Albert N, Waller A, Bringardener S. Economic impact of malnutrition: a model system for hospitalized patients. FPEN 1988; 12: 371-6. 
9 Malone M. Quality of life of patients receiving home parenteral or enteral nutrition support. Pharmacoeconomics 1994; 5: 101-8.

$10 \mathrm{McWhirter}$ JP, Pennington CR. Incidence and recognition of malnutrition in hospital. BMF 1994; 308: 945-8.

11 Kings Fund Centre $A$ positive approach to nutrition as treatment. London: 1992.

12 Payne-James J, de Gara CJ, Grimble GK, et al. Artificial nutrition support in hospitals in the United Kingdom - 1994. Third National Survey (in press). 13 Hamaouri E. Assessing the nutritional support team. JPEN 1987; 11: 412-21.

14 Brown RO, Carlson SD, Cowan GSM, Powers DA, Luther RW. Enteral nutritional support management in a university teaching hospital: team vs non-team. FPEN 1987; 11: 52-7.
15 Faubion WC, Wesley JR, Kharlidi N, Silva J. Total parenteral nutrition catheter sepsis: impact of the team approach. FPEN 1986; 10: 641-5.

16 Traeger S, Williams GB, Milliren G, Young DS, Fisher M, Haug III MT. Total parenteral nutrition by a nutrition support team: improved quality Total parenteral nutrition by a nut

17 Grimble RF. Dietary manipulation of the inflammatory response. Proc Nutr Soc 1992; 51: 285-94

18 Martin R. How nutrition teams affect us - the patients and parents. Birmingham: ESPEN XVI Congress, 1994: Educational Studies 31.

19 Dalton MJ, Schepers G, Gee JP, Alberts CC, Eckhauser FE, Kirking DM. Consultative total parenteral nutrition teams: the effect on the incidence of total parenteral nutrition-related complications. FPEN 1984; 8: $146-52$ 